\section{§13. Geodesic Acoustic Mode Spectroscopy}

Itoh, S.-I., Yagi, M., Inagaki, S. (RIAM, Kyushu Univ.), Itoh, K., Fujisawa, A., Ido, T.,

Sasaki, M., Nagashima, Y. (Univ. Tokyo)

A new kind of plasma diagnostics, geodesic acoustic mode (GAM) spectroscopy, is proposed [1]. Radial eigenmodes of GAMs with discrete eigenfrequencies in toroidal plasmas are analyzed. The eigenmode has the wavelength of the order of $\rho_{i}^{2 / 3} L_{T}^{1 / 3} \quad\left(\rho_{i}\right.$ : ion gyroradius, $L_{T}$ : temperature gradient scale length) and has the highest peak near the radius where the eigenfrequency coincides with the local GAM frequency [2]. Therefore, observing the radial position of the highest peak of eigenmode provides a method to measure the local ion sound velocity. The novel method to measure the ion composition is given, combined with density and temperatures.

The eigenmode propagates in the region where $\omega>\omega_{\mathrm{GAM}}$ holds. In the case where the temperature decreases towards the edge, GAMs are propagating to the edge and evanescent in the inside. The object of this report is to show that the peak of the eigenmode exists near $r=r_{0}$, we neglect the dependence on temperature gradient of the term that is generated by finite gyro radius effect. The eignemode equation has a characteristic scale length, $\lambda=C^{1 / 3} \rho_{i}^{2 / 3} L_{T}^{1 / 3}$, and is normalized as

$$
\frac{\mathrm{d}^{2}}{\mathrm{~d} x^{2}} \phi(x)+x \phi(x)=0
$$

where $x=\left(r-r_{0}\right) \lambda^{-1}$, and $C$ is a numerical coefficient of the order unity. Equation (1) is solved by employing the Airy function:

$$
\phi(x)=\operatorname{Ai}(-x) .
$$

The eigenmode has an inner inflection point at $x=0$, and the peak at $x \simeq 1$. The boundary condition determines the discrete frequency $\omega$, i.e., the resonant radius $r=r_{0}$. The spectrum of GAMs becomes discreet. Therefore, by identifying the peak position of the GAM amplitude (which is located near $r=r_{0}$ ), we can deduce the ion sound velocity at this radius. By observing the radial profiles of eigenmodes at different eigenfrequencies, we are able to deduce the radial profile of the local GAM frequency, $\omega_{\mathrm{G}}\left(r_{0}\right)$.
Thus, the profile of local ion sound velocity, i.e., the profile of effective ion mass by combining temperature profiles, can be deduced.

Figure 1 shows the radial profile of the local GAM frequency (solid line), and thick dots in Fig.1 denote the radial position of the peak of eigenfunction and the eigenfrequency for the $j$-th mode. $(j=1, \ldots$, 10). The thin dashed line shows $\omega_{\mathrm{G}}(r-\lambda)$, which is a shifted form of the local GAM frequency by the amount of $\lambda$, and can be approximated as $\omega_{\mathrm{G}}(r)$, because $r \gg \lambda$ holds. The dots and thin lines show very good agreement. This means that the measurement of the frequency and peak position of amplitude of excited GAM oscillations provides the radial profile of local GAM frequencies. The ion sound velocity at the peak position of GAM oscillation is given by the frequency of the excited mode. If combined with temperature profiles, which are routinely measured in experiments these days, the radial profile of the ion mass can be measured. Once the effective ion mass is obtained, we also obtain the ion concentration ratio. We call this method GAM spectroscopy.

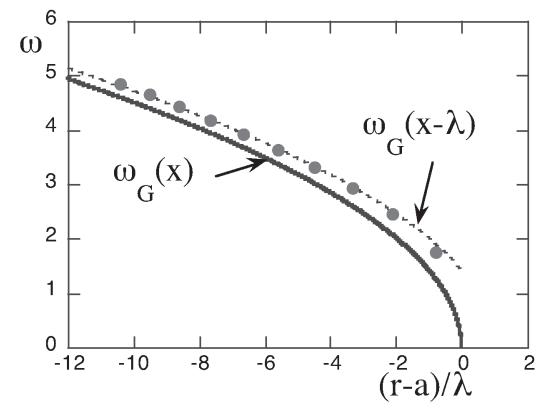

Fig.1 Radial distribution of local GAM frequency (solid line) and the radial location of the peak of eigenfunction with eigenfrequency (thick dots). Thin dashed line indicates the shifted profile of local GAM frequency. The frequency is shown in a unit of $v_{\mathrm{Th} i} / R$ near the left ridge of this figure.

This work was partially supported by Grant-in-Aid for Specially-Promoted Research (16002005) of MEXT and by the collaboration programs of NIFS (NIFS07KOAP017).

[1] Itoh, S.-I. et al.: Plasma Phys. Contr. Fusion 49 (2007) L7-L10

[2] Itoh, K. et al.: Plasma Fusion Res. 1 (2006) 31 